\title{
Pulmonary and aortic endarteritis revealing a patent ductus arteriosus in an adult : a case report
}

\author{
Jaafar Rhissassi ${ }^{1}$, hanae bouhdadi ${ }^{2}$, Hicham Wazaren ${ }^{1}$, Chakib Benlafqih ${ }^{3}$, Rochde Sayah $^{3}$, \\ and Mohammed Laaroussi ${ }^{1}$ \\ ${ }^{1}$ Ibn Sina Hospital \\ ${ }^{2}$ Affiliation not available \\ ${ }^{3}$ Ibn Sina University Hospital Center
}

October 14, 2020

\begin{abstract}
Background The ductus arteriosus, an essential fetal structure, normally closes spontaneously soon after birth. It's persistence into late adulthood is considered rare; infective endarteritis complicating a patent ductus arteriosus (PDA) is an even rarer event. The clinical picture of an infected PDA could be subtle, and the diagnosis is frequently delayed. Case presentation We present the case of a young women who presented with prolonged fever, in whom we made the diagnosis of a patent ductus arteriosus complicated by infective endarteritis with vegetations in both pulmonary and aortic wall with mycotic aneurysms of the descending aorta. She underwent surgery and postoperative course was uneventful. Conclusion To our knowledge, this is the first reported case of a patent ductus arteriosus complicated with both pulmonary and aortic endarteritis.
\end{abstract}

Pulmonary and aortic endarteritis revealing a patent ductus arteriosus in an adult : a case report

Rhissassi, H. Bouhdadi, H. Wazaren, C. Benlafqih, R. Sayah, M. Laaroussi

Cardiovascular surgery department A

Jaafar Rhissassi : Professor of cardiac surgery. First author. Cardiovascular surgery department A. Ibn Sina hospital

Hanae Bouhdadi. MD, Cardiovascular surgery resident. Corresponding author. Cardiovascular surgery department A. Ibn Sina hospital. Email : hanae.bouhdadi@gmail.com.

Adress : Aberrahim Bouabid avenue. Rabat. Morocco

Hicham Wazaren : MD. Cardiovascular surgery resident. Cardiovascular surgery department A. Ibn Sina hospital

Chakib Benlafqih : MD. Cardiovascular surgery. Cardiovascular surgery department A. Ibn Sina hospital

Rochde Sayah : Professor of cardiac surgery. Cardiovascular surgery department A. Ibn Sina hospital

Mohamed Laaroussi : Professer of cardiovascular surgery. Chef of the surgery department. Cardiovascular surgery department A. Ibn Sina hospital

DECLARATIONS

Declarations

Ethical approval and consent to participate : Not applicable 
Consent for publication : The patient gave oral consent for her personal medical report being submitted.

Availability of supporting data : The data set supporting the results of this article is in the manuscript, and included in the reference list.

Competiting interests : Not applicable

Funding : Not applicable

Author's contributions : H. B. received the patient in her consultation and conducted the patient interview.

J.R., C.B and H.B. did the surgery

HB et HW wrote the manuscript

All authors reviewed the final manuscript

Acknowledgments : Not applicable

Abstract

\section{Background}

The ductus arteriosus, an essential fetal structure, normally closes spontaneously soon after birth. It's persistence into late adulthood is considered rare; infective endarteritis complicating a patent ductus arteriosus (PDA) is an even rarer event. The clinical picture of an infected PDA could be subtle, and the diagnosis is frequently delayed.

\section{Case presentation}

We present the case of a young women who presented with prolonged fever, in whom we made the diagnosis of a patent ductus arteriosus complicated by infective endarteritis with vegetations in both pulmonary and aortic wall with mycotic aneurysms of the descending aorta. She underwent surgery and postoperative course was uneventful.

\section{Conclusion}

To our knowledge, this is the first reported case of a patent ductus arteriosus complicated with both pulmonary and aortic endarteritis.

Keys words

Patent ductus arteriosus, infective endarteritis, false aneurysm, case report

\section{Background}

Congenital heart disease (CHD) such as patent ductus arteriosus (PDA) could be a predisposing factor of infective endarteritis (IE).

Mycotic aneurysm is an aneurysm due to an infection of the arterial wall. These are uncommon lesions, with a great potential for hemorrhage or sepsis. Since infectious aneurysm of the aorta has a tendency to expand rapidly in size and rupture, early diagnosis is very important.

We present the case of a 23 year old woman, in whom the diagnosis of patent ductus arteriosus complicated by endarteritis of both pulmonary artery and descending aorta was diagnosed, complicated by mycotic aneurysm of the descending aorta.

After two weeks of antibiotic treatment, the patient underwent surgical removal of the vegetation, closure of the mycotic aneurysm and section suture of the patent ductus arteriosus.

Case presentation 
A 23-year-old female presented with one month history of intermittent high grade fever, associated with lethargy and weight loss (2kg in three weeks).

There was no history of chest pain, hemoptysis, and dyspnea at rest. There was history of recent dental intervention.

On examination, she was febrile and cachectic, with marked conjunctival pallor. There was a continuous murmur, with a thrill over the pulmonary area.

There were no other stigmas of infective endocarditis.

A chest X-ray was unremarkable.

On physical examination, TA was $120 / 80$, heart rate 100 , body temperature was $38,5^{\circ} \mathrm{C}$.

Auscultation revealed a continuous murmur in left 2nd intercostal space.

Laboratory workup showed C-reactive protein $100 \mathrm{mg} / \mathrm{L}$, leukocytes $12,000 / \mathrm{mm} 3$, hemoglobin $8.5 \mathrm{~g} / \mathrm{dL}$

Two consecutive blood were positive for streptococcis viridans.

Transthoracic echocardiography revealed a large PDA $(10 \mathrm{~mm})$ with left to right shunt, and a fixed structure on the wall of the pulmonary artery with erratic movement indicative of a vegetation (Figure 1) and mobile vegetation attached to the wall of the descending aorta in the supra sternal view (Figure 2), a left ventricle with conserved systolic function and $55 \mathrm{~mm}$ end-diastolic diameter

Computed tomography (CT) scan showed two mycotic pseudoaneurysms of the descending aorta (Figure 3).

Following three days of antibiotic treatment (ampicillin and gentamycin), she had symptomatic improvement and became afebrile.

A repeat echocardiography did show a disparition of the pulmonary vegetation, a repeated CT scann showed no embolism to the pulmonary trunk.

Decision was made for surgical intervention.

After establishment of a femoro femoral partial by-pass. The thorax was entered through the left fourth intercostal space. Two juxta- ductal aneurysmal formations with inflammatory adhesions were found (Figure $4)$.

The proximal clamp was placed across the left subclavian artery and the distal clamp inferior to the false aneurysms site. The ductus arteriosus was initially clamped.

The descending aorta was opened, we proceeded to debridement of the infected aorta from the vegetations and we decided to close the false aneurysms by direct sutures (Figure 5).

We then proceeded to a section suture of the patent ductus arteriosus.

Postoperative course was uneventful.

Patient was discharged home after two weeks, she was asymptomatic at 3-months of follow-up.

Discussion

PDA accounts for $5 \%$ to $10 \%$ of all congenital heart disease. [1]

A PDA that persists beyond 1 month of age is estimated to occur in $0.3-0.8-4$ per 1000 live births. [2]

The size of a PDA can range from very large to $<1 \mathrm{~mm}$, and accordingly, the clinical findings associated with a PDA can vary considerably.

Infective endarteritis (IE) was the most common cause of death in patients with patent ductus arteriosus (PDA) prior to the introduction of antibiotic therapy and surgical closure of the defect, though nowadays it 
is a rare complication. Infective endarteritis is especially unusual in asymptomatic patients, more so when the PDA is silent on cardiac auscultation, with very few reports of this type of case to be found in the relevant literature. [3]

An unrepaired PDA is a risk factor for IE, and when it occurs, vegetations usually occur on the pulmonary artery end of the ductus; embolic events are usually of the lung rather than the systemic circulation. [4]

The proposed pathogenesis of IE associated with congenital heart disease involves complex interactions among the damaged valvular or mural endocardium exposing the matrix proteins to thromboplastin and tissue factor, formation of a nonbacterial thrombotic endarteritis, and eventual microbial adherence, colonization, and replication. The turbulent blood flow through the aorta and pulmonary artery which causes endothelial injury and subsequent seeding of pathogens onto the injured endothelium is central to the proposed pathogenetic mechanisms for the development of vegetations particularly in patients with PDA. [5]

The management of mycotic aneurysm is unclear. There are no strategies or recommendations regarding the timing of surgery (before or after antibiotics), aortic reconstruction (with or without material), and the duration of post-operative antibiotic therapy. Studies suggest that combined medical and surgical treatment decreases mortality. [6]

\section{Conclusion}

In conclusion, we hereby report an unusual case of a young lady who had infective endarteritis secondary to unknown PDA.

As the case reported shows, the risk for infection is present even in asymptomatic ducts, and prophylactic closure of the PDA should be considered

Abbreviations

PDA : Patent ductus arteriosus

CHD : Congenital heart disease

IE : Infective endarteritis

CT : Computed tomography

\section{References}

1. Hoffman JI, Kaplan S. The incidence of congenital heart disease. J Am Coll Cardiol. 2002;39:1890-900.

2. Schneider DJ, Moore JW (2006) Patent ductus arteriosus. below Circulation 114: 1873-1882.

3. Parthenakis F, Kanakaraki M, Vardas P. Silent patent ductus arteriosus endarteritis. Heart, 84 (2000), pp. 619

4. John Fani Srour. Infective Endarteritis in a Patent Ductus Arteriosus. Srour. Int J Respir Pulm Med $2014,1: 1$

5. Choi K. N., Yang T. H., Park B. S., et al. A case with patent ductus arteriosus complicated by pulmonary artery endarteritis. Journal of Cardiovascular Ultrasound. 2008;16(3):90-92.

6. Choi M, Mailman TL. Pneumococcal endocarditis in infants and children. Pediatr Infect Dis J 2004;23:166-171

Figure legends

Figure 1: Large PDA and a fixed structure on the wall of the pulmonary artery

Figure 2 : Supra sternal view : vegetation attached to the wall of the descending aorta

Figure 3 : Computed tomography scan showing two mycotic pseudoaneurysm of the descending aorta

Figure 4 : Peroperative image showing two juxta-ductal aneurysmal formations 
Figure 5 : Peroperative image after opening of the descending aorta. Left : Vegetations on the wall of the aorta. Right : closure of the false aneurysms by direct sutures
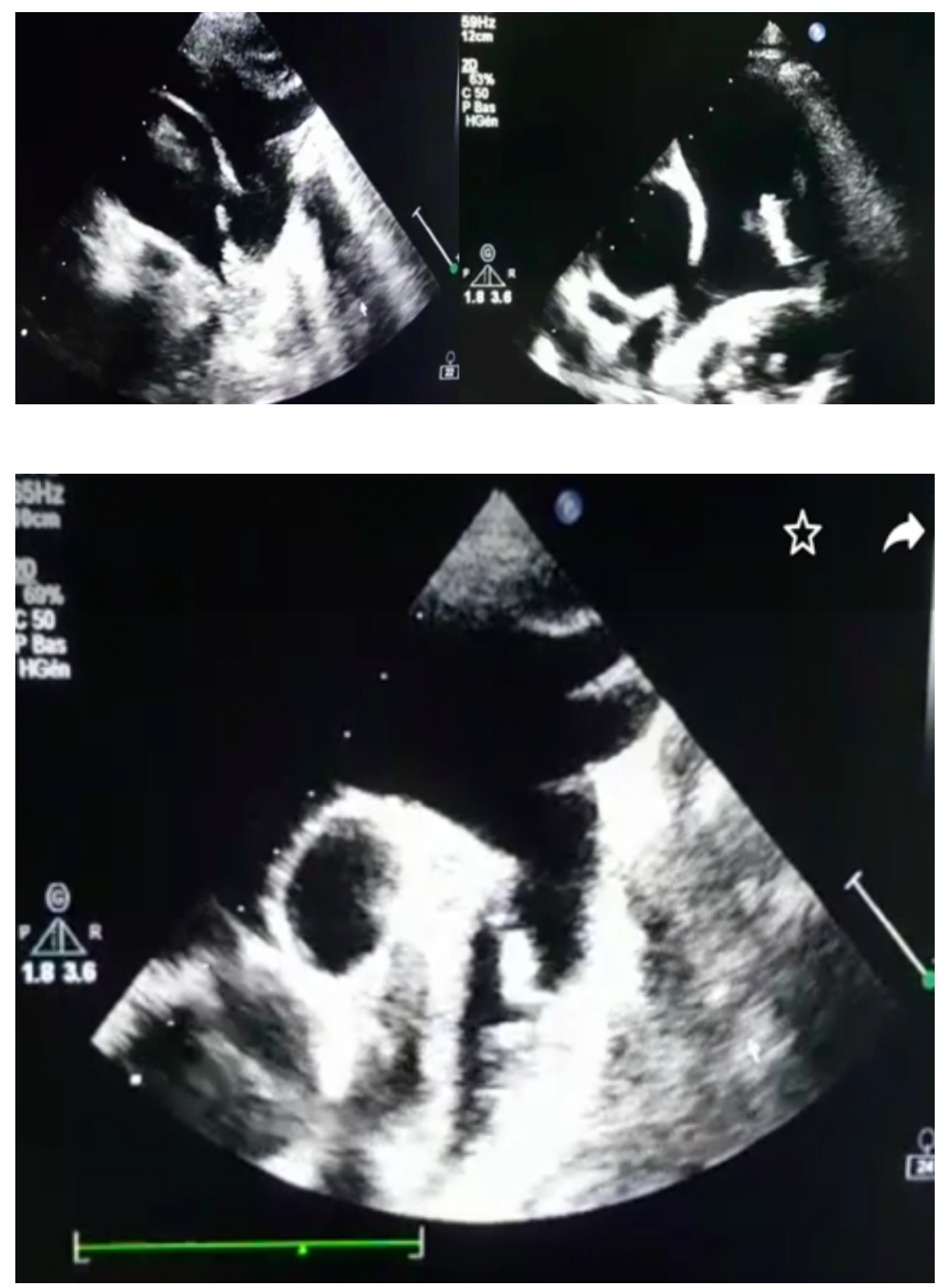

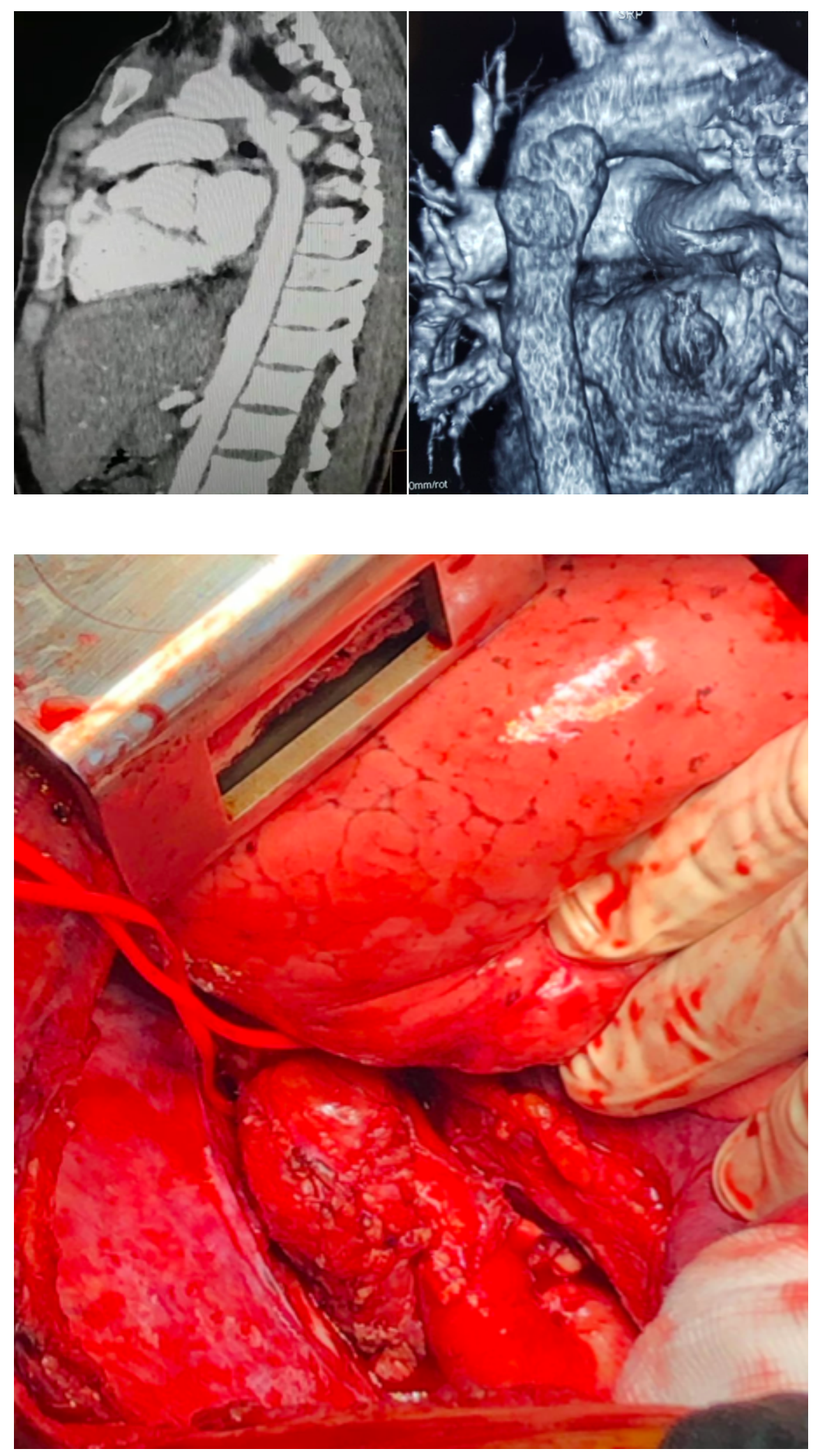


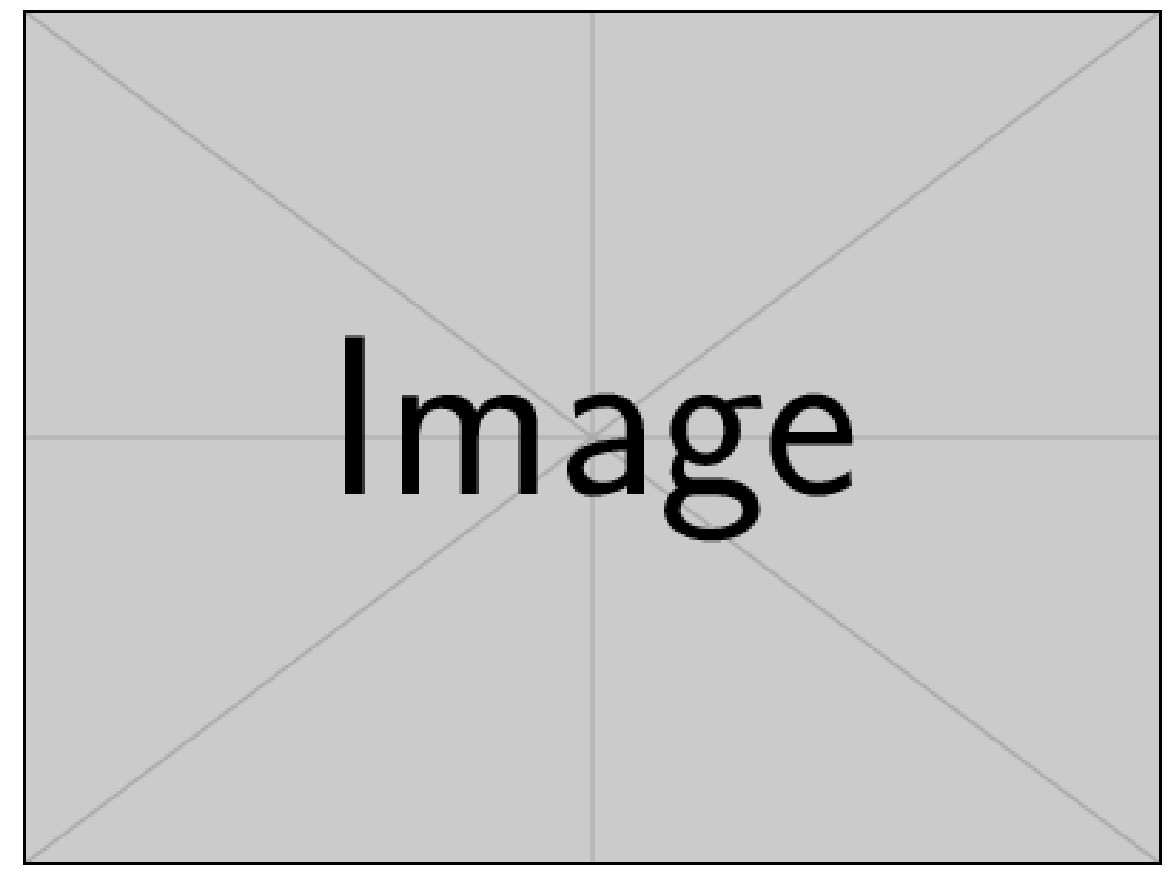

